\title{
44544 - PREOPERATIVE HYPERGLYCEMIA - A NEW RISK FACTOR FOR PULMONARY EMBOLISM
}

Disclosure: Grants/research support - Johnson \& Johnson LifeScan grant. No 08010000-A91401

\author{
Boris Mraovic, Thomas Jefferson University, Philadelphia, PA, USA; \\ JEFFREY JOSEPH, THOMAS JEFFERSON UNIVERSITY; \\ ZVI GRUNWALD, THOMAS JEFFERSON UNIVERSITY; \\ J PARVIZI, THOMAS JEFFERSON UNIVERSITY; \\ B HIPSZER, THOMAS JEFFERSON UNIVERSITY; \\ L PULIDO, THOMAS JEFFERSON UNIVERSITY; \\ C RESTREPO, THOMAS JEFFERSON UNIVERSITY;
}

INTRODUCTION: Diabetes mellitus (DM) is a hypercoagulable state.1 A longitudinal study of 19,293 men and women found that DM increased the risk for DVT and/or PE2. But DM was not found to be a significant independent risk factor for thromboembolic events following total joint arthroplasty. 3 Healthy young men with induced hyperglycemia and hyperinsulinemia developed an elevation in plasma coagulation factors associated with thrombus formation.4 It is unknown whether hyperglycemia is an independent risk factor for developing PE after major orthopedic surgery. We investigated if preoperative hyperglycemia was associated with PE in patients undergoing total joint arthroplasty.

METHODS: After obtaining IRB approval, we reviewed the medical records of patients undergoing total hip or total knee replacement from January 2001 to April 2006. Patients with repeated surgeries or without preoperative glucose values were excluded from the study. The diagnosis of PE was based on a positive finding with a chest CT or lung scan. Patients were divided into 4 groups based on preoperative blood glucose (BG) levels: low normal BG < 70 $\mathrm{mg} / \mathrm{dl}$, normal 70-125 mg/dl, high 126-199 mg/dl, and very high \&\#8805;200 mg/dl. Data are mean \pm SD and were analyzed using the Fisher's exact test and the exact version of CochranArmitage Trend Test.

RESULTS: A total of 7377 patients who underwent joint replacement were included in the study. BG was low normal in 110 patients, normal in 6213 patients, high in 906 patients, and very high in 148 patients. Patients underwent surgery $15.7 \pm 8.3$ days after the preoperative clinic visit. All patients received postoperative DVT prophylaxis with warfarin per hospital protocol. There was a significant correlation between the preoperative BG and the incidence of postoperative PE ( $\mathrm{p}=0.003)$. The incidence $(95 \% \mathrm{CI})$ of PE was: $0 \%(0-3.3 \%)$ in the low normal group, $1.45 \%(1.17-1.78 \%)$, in the normal group, $1.66 \%(0.93-2.72 \%)$ in the high group and $5.41 \%(2.36-10.4 \%)$ in the very high glucose group. Preoperative BG \&\#8805;200mg/dl increased the incidence of PE by a factor of $3.9(1.3$ - 8.7) when compared with patients in the normal BG group, and by $3.2(1.4-7.3 \%)$ when compared with patients in the high BG group. Of interest, nobody in the low normal glucose group developed PE.

DISCUSSION: Our data suggests that preoperative hyperglycemia \& $\# 8805 ; 200 \mathrm{mg} / \mathrm{dl}$ is a risk factor for developing PE after total joint replacement. A prospective controlled study is needed 
to determine whether tight control of preoperative glucose levels leads to a decreased incidence of PE in this clinical setting, and what target glycemia level should be before mayor orthopedic surgery.

REFERENCES:

1. J Diabetes Complications 2001 15: 44-54

2. Arch Intern Med 2002 162: 1182-89

3. Anesthesiology 2003 99: 552-60

4. Diabetes 1999 48: 1156-61 